\title{
Non-invasive stroke volume assessment in patients with pulmonary arterial hypertension: left-sided data mandatory
}

\author{
Gert-Jan Mauritz1, J Tim Marcus², Anco Boonstra1, Pieter E Postmus1, \\ Nico Westerhof 1,3 and Anton Vonk-Noordegraaf*1
}

\begin{abstract}
Address: ${ }^{1}$ Department of Pulmonary Diseases, Institute for Cardiovascular Research ICaR-VU, VU University Medical Center, Amsterdam, The Netherlands, ${ }^{2}$ Department of Physics and Medical Technology, Institute for Cardiovascular Research ICaR-VU, VU University Medical Center, Amsterdam, The Netherlands and ${ }^{3}$ Department of Physiology, Institute for Cardiovascular Research ICaR-VU, VU University Medical Center, Amsterdam, The Netherlands

Email: Gert-Jan Mauritz - gj.mauritz@vumc.nl; J Tim Marcus - jt.marcus@vumc.nl; Anco Boonstra - a.boonstra@vumc.nl; Pieter E Postmus - pe.postmus@vumc.nl; Nico Westerhof - n.westerhof@vumc.nl; Anton Vonk-Noordegraaf* - a.vonk@vumc.nl

* Corresponding author
\end{abstract}

Published: 5 November 2008

Journal of Cardiovascular Magnetic Resonance 2008, 10:5 I doi:10.1186/1532-429X-10-51

This article is available from: http://www.jcmr-online.com/content//0/I/5 I

(C) 2008 Mauritz et al; licensee BioMed Central Ltd.

This is an Open Access article distributed under the terms of the Creative Commons Attribution License (http://creativecommons.org/licenses/by/2.0), which permits unrestricted use, distribution, and reproduction in any medium, provided the original work is properly cited.
Received: 18 August 2008

Accepted: 5 November 2008

\begin{abstract}
Background: Cardiovascular Magnetic Resonance (CMR) is an emerging modality in the diagnosis and follow-up of patients with Pulmonary Arterial Hypertension (PAH). Derivation of stroke volume (SV) from the pulmonary flow curves is considered as a standard in this respect. Our aim was to investigate the accuracy of pulmonary artery (PA) flow for measuring SV.

Methods: Thirty-four PAH patients underwent both CMR and right-sided heart catheterisation. CMR-derived SV was measured by PA flow, left (LV) and right ventricular (RV) volumes, and, in a subset of nine patients also by aortic flow. These SV values were compared to the SV obtained by invasive Fick method.

Results: For SV by PA flow versus Fick, $r=0.7 \mathrm{I}$, mean difference was $-4.2 \mathrm{ml}$ with limits of agreement 26.8 and $-18.3 \mathrm{ml}$. For SV by LV volumes versus Fick, $r=0.95$, mean difference was -0.8 $\mathrm{ml}$ with limits of agreement of 8.7 and $-10.4 \mathrm{ml}$. For SV by RV volumes versus Fick, $r=0.73$, mean difference $-0.75 \mathrm{ml}$ with limits of agreement 21.8 and $-23.3 \mathrm{ml}$. In the subset of nine patients, SV by aorta flow versus Fick yielded $r=0.95$, while in this subset $S V$ by pulmonary flow versus Fick yielded $r=0.76$. For all regression analyses, $p<0.0001$.
\end{abstract}

Conclusion: In conclusion, SV from PA flow has limited accuracy in PAH patients. LV volumes and aorta flow are to be preferred for the measurement of SV.

\section{Introduction}

In pulmonary arterial hypertension (PAH), cardiovascular magnetic resonance (CMR) has been proposed as a standard for the assessment of right ventricular function and characteristics of the pulmonary vascular bed $[1,2]$. Accurate assessment of stroke volume (SV) by CMR is critical in this respect, since earlier studies revealed that SV is closely related to prognosis and that a change in SV reflects treatment effects $[3,4]$. Since most of the CMR protocols used in PAH $[5,6]$ measured pulmonary artery flow, SV can be assessed by measuring flow in the main pulmonary artery (PA). 
Previous studies have shown that this method is accurate to measure SV from PA flow in healthy subjects [7-9]. Whether this also holds true in PAH is questionable, since the velocity profile in PAH is non-laminar, in contrast to the profile in healthy subjects [10-13].

For this test of accuracy, a clinical standard is required. This standard is provided by the measurement of SV by the direct Fick principle during right heart catheterisation (RHC) [14]. However, this is an invasive procedure and thus not well suited for either screening or frequently repeated follow-up measurements.

Therefore the aim of the present study is to assess the accuracy of the PA flow by CMR for measuring SV in PAH patients, by comparing SV from this PA flow with the SV assessed by the Fick method. In addition, other CMRderived SV measures from aorta flow and cine imaging in the same patients will also be compared versus the Fickderived SV.

\section{Materials and methods Patients}

This study was approved by the institutional Review Board on Research Involving Human Subjects of the VU University medical centre, and all participants gave written informed consent. Between January 2004 and April 2007, a total of 34 patients who were given a final diagnosis of PAH after a complete diagnostic workup including RHC, underwent CMR. RHC and CMR were performed within 12 hours. The study group consisted of 23 female (68\%) and 11 male (32\%) patients with a mean age of 45 years \pm 17 (standard deviation), and an age range of 20 84 years.

\section{CMR imaging protocol}

\section{$C M R$ flow measurements}

SV was measured using both phase contrast flow and volumetric methods as described below.

CMR was perforned with a Siemens $1.5 \mathrm{~T}$ 'Sonata' whole body scanner (Siemens Medical Solutions, Erlangen. Germany), equipped with a phased-array body coil.

Phase-contrast CMR was acquired during continuous breathing with a gradient echo MR sequence, with velocity encoding perpendicular to the imaging plane and a velocity sensitivity of $120 \mathrm{~cm} / \mathrm{sec}$. The flow sequence was run with the following parameters: orientation = orthogonal to the main PA, slice thickness $=6 \mathrm{~mm}$, field of view $=240$ $\times 320 \mathrm{~mm}^{2}$, matrix size $=140 \times 256$, echo time $=4.8 \mathrm{~ms}$, repetition time $=11 \mathrm{~ms}$, temporal resolution $=22 \mathrm{~ms}$, flip angle $=25^{\circ}$.
To explore whether there is inaccurate determination of flow-derived SV due to inherent technical limitations of phase-contrast CMR, aortic flow was also measured in a subset of nine patients, approximately $2-4 \mathrm{~cm}$ above the aortic valve and distal to the coronary arterial ostia, and the aortic flow-derived SV was also compared to the Fickderived SV.

After the flow images were acquired, a 7 litre bottle containing $\mathrm{H} 2 \mathrm{O}$, with per litre $1.25 \mathrm{~g}$ NiSO $4.6 \mathrm{H} 2 \mathrm{O}+2.6 \mathrm{~g}$ $\mathrm{NaCL}$ ("phantom") was then imaged with identical imaging parameters, to serve as correction for the background phase error in the mean PA and Aorta[15].

\section{$R V$ and $L V$ volumetric measurements}

The short-axis slices needed to encompass the entire left and right ventricle volumes to measure right ventricular $(\mathrm{RV})$ and left ventricular (LV) volumes-derived SV, were obtained by steady state free precession imaging: 11 phase encoding lines per heart beat (which means 11 lines per segment), flip angle $=60^{\circ}$, slice thickness $=6 \mathrm{~mm}$, slice gap $=4 \mathrm{~mm}$, temporal resolution $36.3 \mathrm{~ms}$ and retrospectively ECG gated. Acquisition was in breathhold, acquisition time was 14 heartbeats.

\section{Imaging analysis \\ CMR flow measurements}

CMR post-processing was performed using the 'FLOW' software package (Dept. of Radiology, Leiden University Medical Center, Leiden, The Netherlands). The heart rate during cardiac CMR was recorded from the images. No aliasing due to high peak systolic velocities was encountered. The contours of the mean pulmonary artery MPA were automatically traced, with manual correction when necessary, simultaneously on magnitude and velocitymap images of all reconstructed phases. The software then calculated the velocity in each of the pixels included within the contours. The flow in each pixel (velocity times pixel area) is calculated and the pixel flow within the contour is summed. This is done for every temporal phase, resulting in blood flow as a function of time through the main PA.

Following image acquisition, pulmonary blood flow was corrected using the offset values from the phantom (figure 1). After background correction with the phantom (figure 2), we calculated PA forward flow volume as the area under the curve until the zero-crossing of the downward limb. We checked whether there was more than 5\% reverse flow that would indicate any pulmonary regurgitation (PR). However we did not observe any PR, thus we did not exclude any PR patient. The same was done for the Aorta flow. 

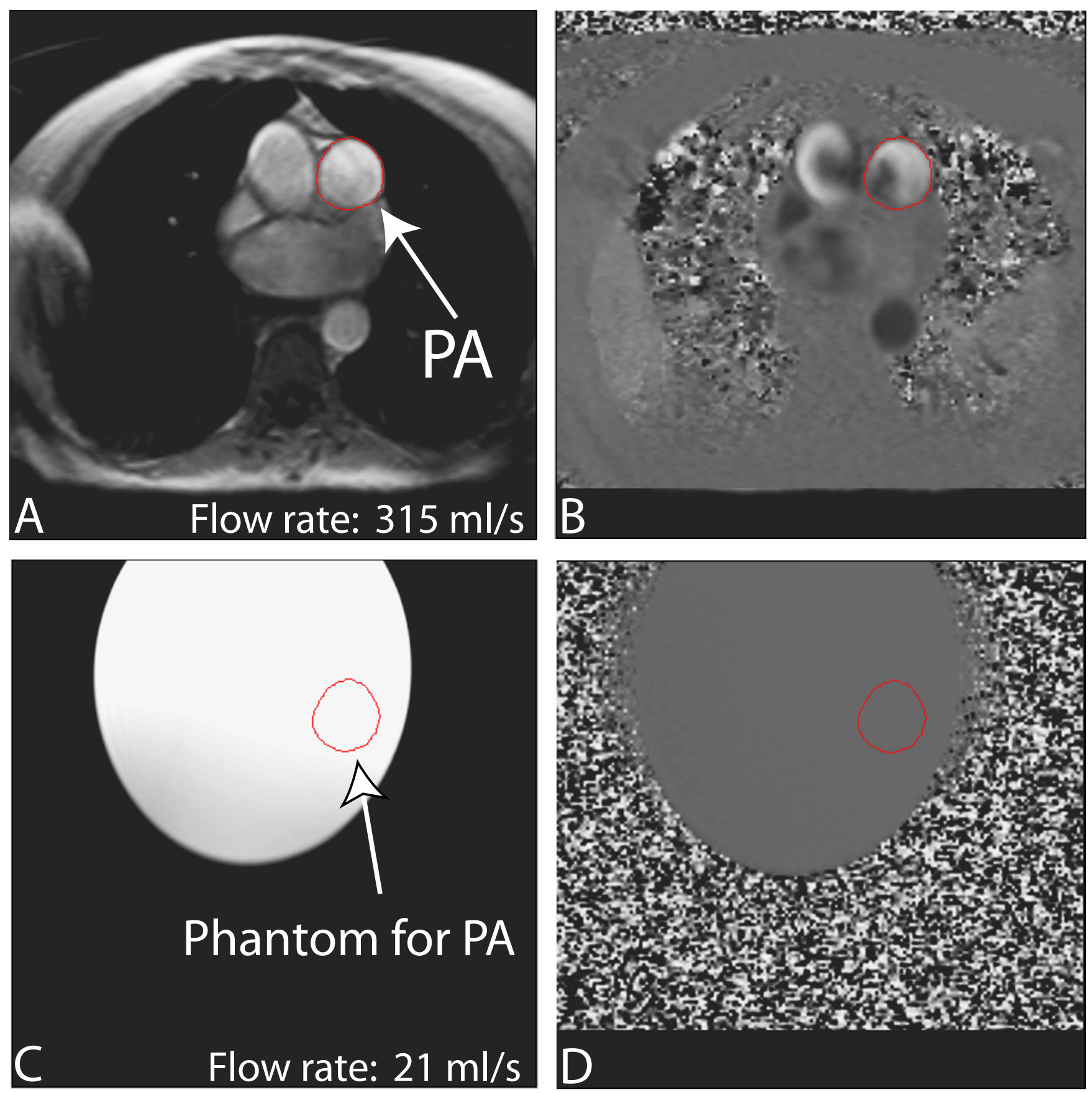

Figure I

Double-oblique gradient-echo phase-contrast CMR (repetition time ms/echo time msec I I/4.8; flip angle 25 deg; section thickness, $6 \mathrm{~mm}$; matrix, $140 \times 256$ ), perpendicular to pulmonary trunk. Region of interest (ROI) placement is shown for measuring pulmonary artery (PA) flow. In (a) magnitude and (b) velocity image is shown. The images correspond to the time frame when maximum flow is measured in the PA. ROI placement for the corresponding phantom magnitude (c) and velocity (d) image is shown to correct velocity offset error in PA. 


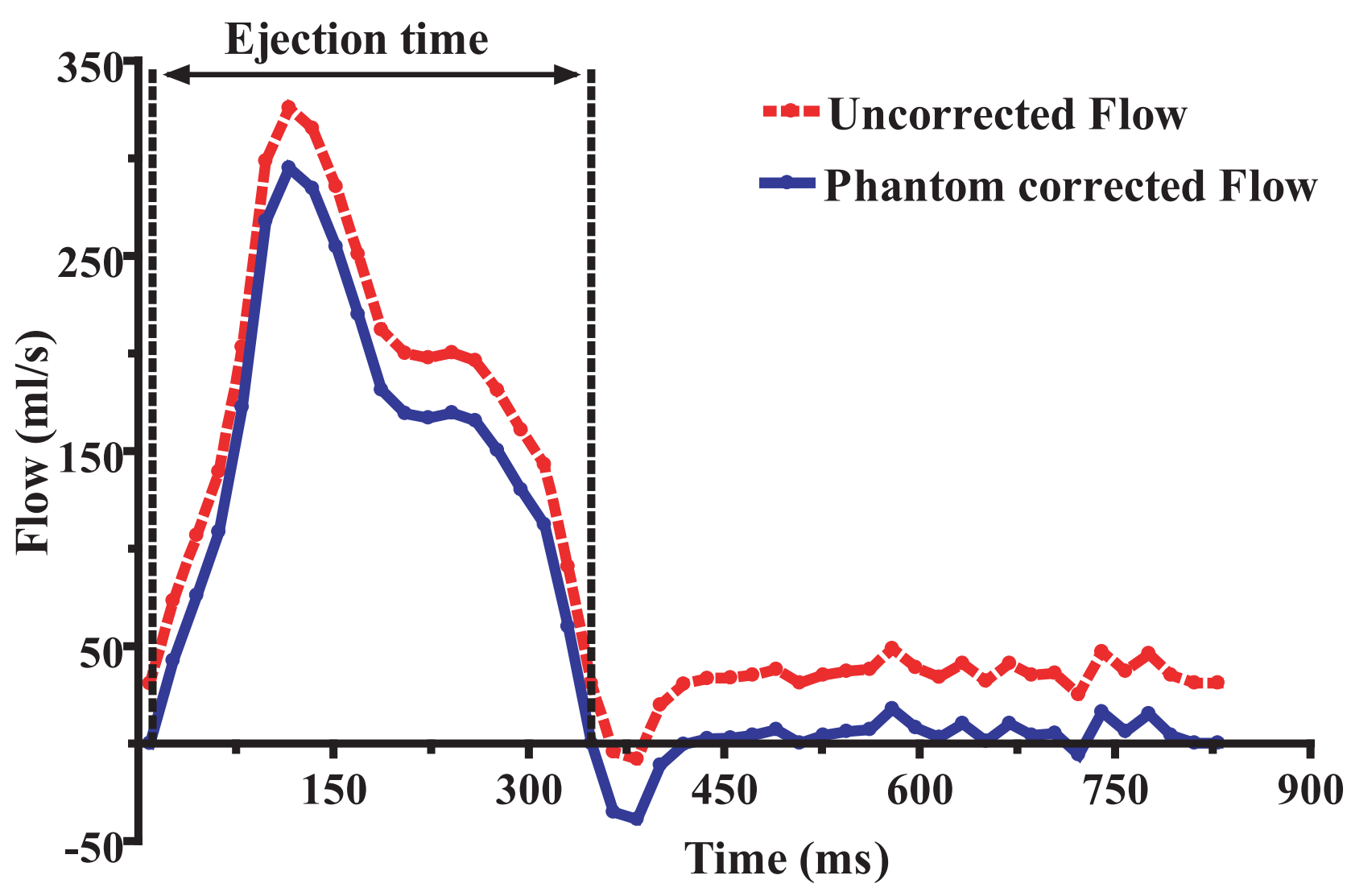

Figure 2

Flow curve of the main pulmonary artery (MPA) during a cardiac cycle in a PAH patient. Uncorrected stroke volume (SV) is obtained by integration of the flow curve during ejection time and is $58 \mathrm{ml}$, ejection time is $0.323 \mathrm{~s}$ and phantom offset value is $21.13 \mathrm{ml} / \mathrm{s}$. Phantom corrected SV is $(58-(0.323 * 21.13))=51 \mathrm{ml}$.

\section{$R V$ and $L V$ volumetric measurements}

We measured RV and LV volumes as follows [16]. The endocardial contours of the ventricles were manually traced on short-axis slices in end-diastolic (first cine phase of the R-wave triggered acquisition) and end-systolic (image phase with smallest cavity area) phases using commercial software (MASS software package, version 5.0,) (figure 3). In the present study, papillary muscles were excluded from manual tracings of the endocardial contours of the right end left ventricle. The ventricular areas were then measured and ventricular volumes calculated by adding the ventricular areas and multiplying by the slice distance. End-Diastolic Volumes (EDV) and EndSystolic Volumes (ESV) were used to calculate LV-and RV volumes-derived SV. One investigator analyzed both phase contrast Flow and volumetric images unaware of stroke volume values measured by direct Fick principle.

\section{Right Heart Catheterization (RHC)}

Diagnostic right heart catheterization was performed with a balloon tipped, flow directed 7F Swan-Ganz catheter (131HF7; Baxter Healthcare Corp; Irvine, CA). The direct
Fick Principle (DF) was used. The patient was in stable condition, lying supine and breathing room air. Right atrial, right ventricular, pulmonary artery and pulmonary capillary wedge pressures were measured. Heart rate was monitored continuously. Average steady state oxygen consumption was obtained at the same time during RHC using a timed collection of expired air to measure oxygen consumption by an on-line analyzer (Vmax 229, Sensormedics, Yorba Linda, USA) connected to a mouth piece. To ensure accuracy, the system was calibrated before each study and the values were time-averaged over at least 5 minutes. Simultaneous arterial and mixed venous blood samples were then drawn for measurement of arterial oxygen saturation $\left(\mathrm{SaO}_{2}\right)$ and haemoglobin $(\mathrm{Hb})$ concentration. Arterial blood samples were obtained through either a radial or femoral arterial puncture and the mixed venous blood samples were obtained from the distal port of the pulmonary artery catheter. The cardiac output (CO) was then calculated by dividing the average oxygen consumption $\left(\mathrm{VO}_{2}\right)$ value with the difference between the concentration of arterial oxygen $\left(\mathrm{CaO}_{2}\right)$ and concentration of mixed venous oxygen content $\left(\mathrm{CvO}_{2}\right)$. Eventually, SV 

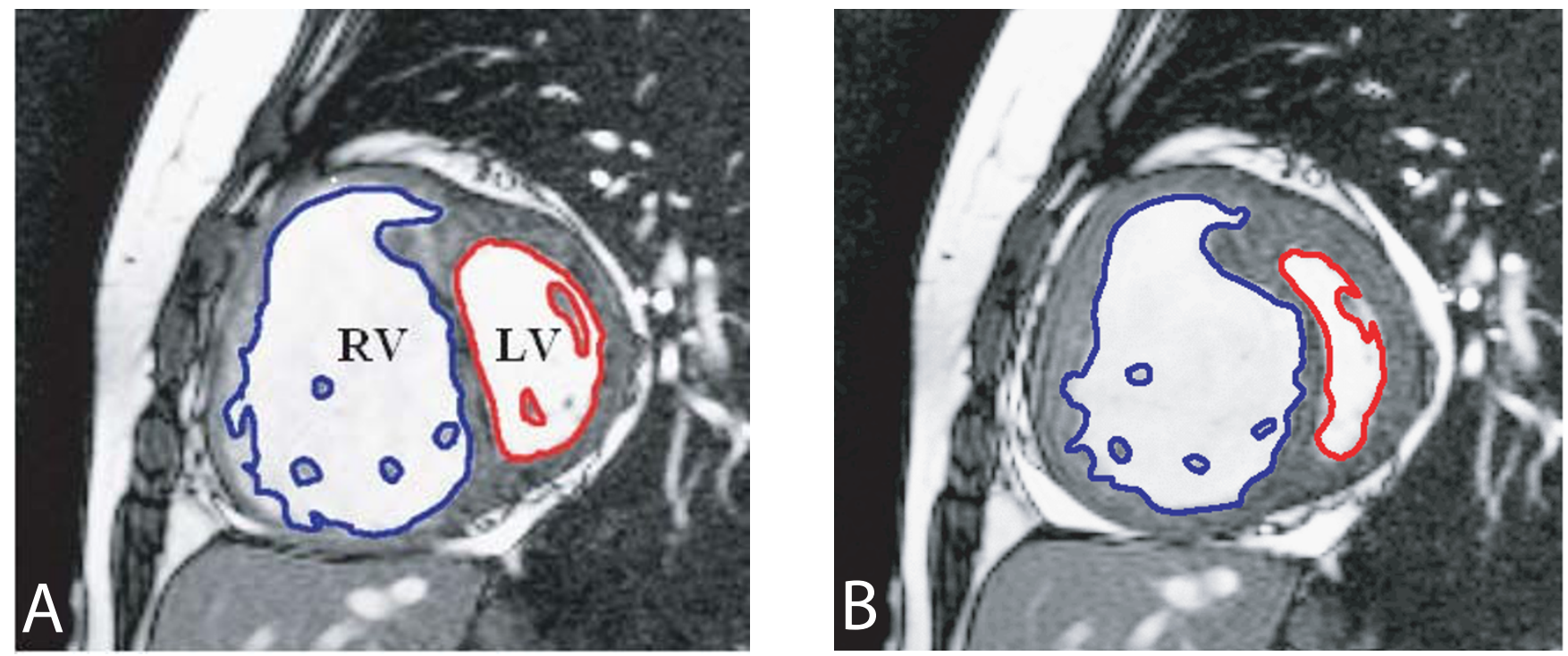

Figure 3

Double-oblique steady-state free precession cine MR images (repetition time ms/echo time ms 3.3/I.65; flip angle 60 deg; section thickness, 6 mm; matrix, $256 \times$ I50). End-diastolic (a) and end-systolic (b) cardiac short axis slice in PAH patient. The endocardial boundaries of the right ventricle (RV) and left ventricle (LV) are traced for calculation of enddiastolic volume (EDV) and end-systolic volume (ESV). EDV and ESV were calculated by summation of the product (area $\times$ slice distance) for all slices. SV is then given by SV = EDV-ESV for RV and LV.

(ml) was determined by dividing $\mathrm{CO}(\mathrm{L} / \mathrm{min})$ by $\mathrm{HR}$ (beats/min).

\section{Intra-observer and inter-observer variability}

Interobserver variability of the different CMR methods was assessed by a second investigator analyzing all of data sets. Intraobserver variability was analyzed by assessing SVs obtained by the different CMR methods twice by one observer. The two assessments were separated by a one month period, and the observer was blinded for his previous results.

\section{Data analysis}

The results are expressed as mean \pm SD. For each measurement of SV, the values for SV obtained invasively direct Fick and by the CMR-based methods were compared by regression analysis. Bland-Altman analysis was used to compare the degree of agreement between the three CMR methods and the Fick method for SV measurement [17]. Bias was defined as the mean value of the differences between CMR methods and the Fick principle. Precision was defined as 1 standard deviation (SD) of the differences and limits of agreement as the bias \pm 2 SDs reported as millilitres. The percentage error was calculated as the ratio of 2 times the SD to mean SV. Tendency toward overor underestimation of SV by the different CMR-based methods was assessed with the two-tailed Student's $t$-test for paired data.
The mean difference (bias) and the coefficient of variability $(\mathrm{CoV}=\mathrm{SD}$ of repeated measures as $\%$ of their mean $)$ were used to assess intra- and interobserver variability.

$P$ values less than .05 were considered to indicate significant differences. All statistical analyses were performed using GraphPad PRISM (version 4.0, GraphPad software, San Diego, CA).

\section{Results}

The demographic data, causes of PAH and resting clinical hemodynamic measurements obtained at RHC of the 34 PAH patients are shown in Table 1. Twenty-nine patients (out of 34) had measurable Tricuspid Regurgitation by echo, and 4 patients had right-to-left shunting based on the passage of contrast bubbles through a patent foramen ovale, observed by echo-cardiography.

Figure 4 shows the results of the three different CMR methods to measure stroke volume in comparison with SV measurements assessed by the direct Fick principle. From this figure it is clear that the PA flow-derived SV values and the RV volumes-derived SV values show only a limited correlation with the Fick standard. LV volumesderived SV showed the best correlation with Fick.

Figure 5 shows the Bland-Altman plots of the difference between the CMR based methods and the direct Fick against the mean of both values. Mean $( \pm$ SD), Mean Dif- 
Table I: Baseline characteristics of the patients*

\begin{tabular}{|c|c|}
\hline Characteristic & Value \\
\hline No of PAH patients & $\mathrm{n}=34$ \\
\hline Idiopathic PAH & $\mathrm{n}=14$ \\
\hline Familial PAH & $n=6$ \\
\hline $\mathrm{PAH}$ and Collagen vascular disease & $\mathrm{n}=5$ \\
\hline $\mathrm{PAH}$ and HIV infection & $\mathrm{n}=6$ \\
\hline $\mathrm{PAH}$ and Porto pulmonary syndrome & $\mathrm{n}=3$ \\
\hline Age, $y r$ & $54 \pm 17$ \\
\hline Male/female & $11 / 23$ \\
\hline \multicolumn{2}{|l|}{ Functional status: } \\
\hline NYHA II & $n=18$ \\
\hline NYHA III & $\mathrm{n}=12$ \\
\hline NYHA IV & $\mathrm{n}=4$ \\
\hline 6 min walk test, $\mathrm{m}$ & $441 \pm 109$ \\
\hline \multicolumn{2}{|l|}{ Hemodynamic variables: } \\
\hline Pra, $\mathrm{mmHg}$ & $7 \pm 4.5$ \\
\hline sPap, $\mathrm{mmHg}$ & $74 \pm 21$ \\
\hline $\mathrm{dPap}, \mathrm{mmHg}$ & $29 \pm 11$ \\
\hline $\mathrm{mPap}, \mathrm{mmHg}$ & $45 \pm 10$ \\
\hline Pcwp, $\mathrm{mmHg}$ & $6.9 \pm 3.3$ \\
\hline $\mathrm{SaO}_{2}, \%$ & $95 \pm 2$ \\
\hline $\mathrm{SvO}_{2}, \%$ & $63 \pm 8$ \\
\hline Q-Fick, I/min & $4.7 \pm 1.3$ \\
\hline $\mathrm{Cl}, \mathrm{I} \cdot \mathrm{min}^{-1} \cdot \mathrm{m}^{-2}$ & $2.5 \pm 0.6$ \\
\hline $\mathrm{HR}, \min ^{-1}$ & $81.5 \pm 12$ \\
\hline SV-Fick, $\mathrm{ml} /$ beat & $58.8 \pm 16$ \\
\hline PVR, dynes $\cdot \mathrm{s}^{-1} \cdot \mathrm{cm}^{-5}$ & $777 \pm 402$ \\
\hline
\end{tabular}

$\mathrm{PAH}=$ idiopatische pulmonary arterial hypertension; NYHA = modified New York Association; Pra = right atrial pressure; $\mathrm{sPap}=$ systolic pulmonary artery pressure; $\mathrm{dPap}=$ diastolic pulmonary artery pressure; $\mathrm{mPap}=$ mean pulmonary artery pressure; $\mathrm{Pcwp}=$ pulmonary capillary wedge pressure; $\mathrm{SaO} 2=$ arterial oxygen saturation $\mathrm{SvO}_{2}=$ mixed venous oxygen saturation; Q-Fick = cardiac output (as estimated by the Fick method; $\mathrm{Cl}=$ cardiac index; $\mathrm{HR}=$ heart rate; SV-Fick = stroke volume PVR = pulmonary vascular resistance;

$*$ Values are expressed as mean $\pm S D$

ference (bias), SD of the difference (precision), limits of agreement, and percentage error according to different CMR based SV measurements are presented in Table 2. As shown in this Table 2, the limits of agreement of the PA flow-derived SV with Fick present almost the same range of dispersion as was found when RV volumes-derived SV was compared with Fick. The limits of agreement between Fick and LV volumes-derived SV are narrow.

Bland-Altman analysis demonstrated a small degree of underestimation of SV by PA flow with mean difference of $4.2 \mathrm{ml}$, the underestimation was statistically significant (P $=0.039$ ).

If those patients with echo-derived right-to-left shunting were excluded, than the correlation coefficient between PA Flow-derived SV and Fick-derived SV increased to $\mathrm{r}^{2}=$ 0.56 and the bias decreased to a non-significant level of $1.9 \pm 3.7 \mathrm{ml}$.
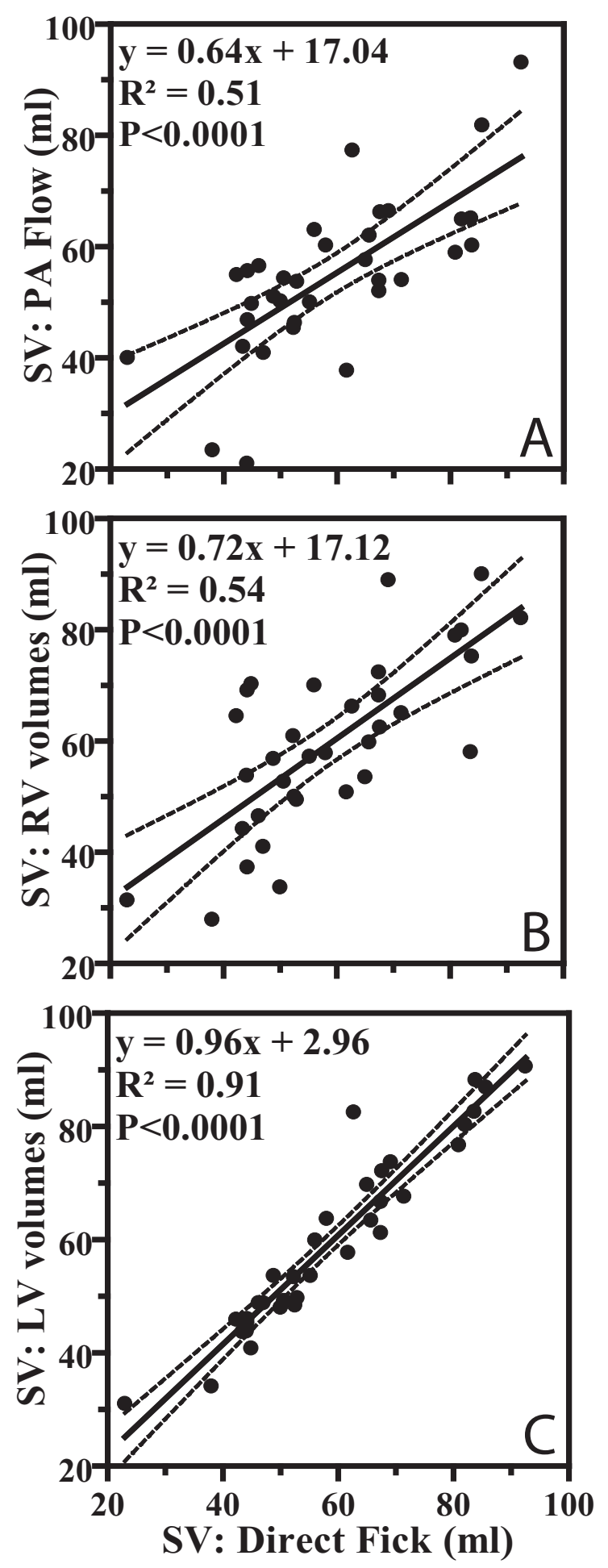

Figure 4

Linear regression analysis of the correlation between direct Fick and (a) PA flow with phase-contrast MRI, (b) RV volumetric assessment and (c) LV volumetric assessment in 34 PAH patients for stroke volume (SV) (ml). Dashed lines $=95 \%$ Cls. 


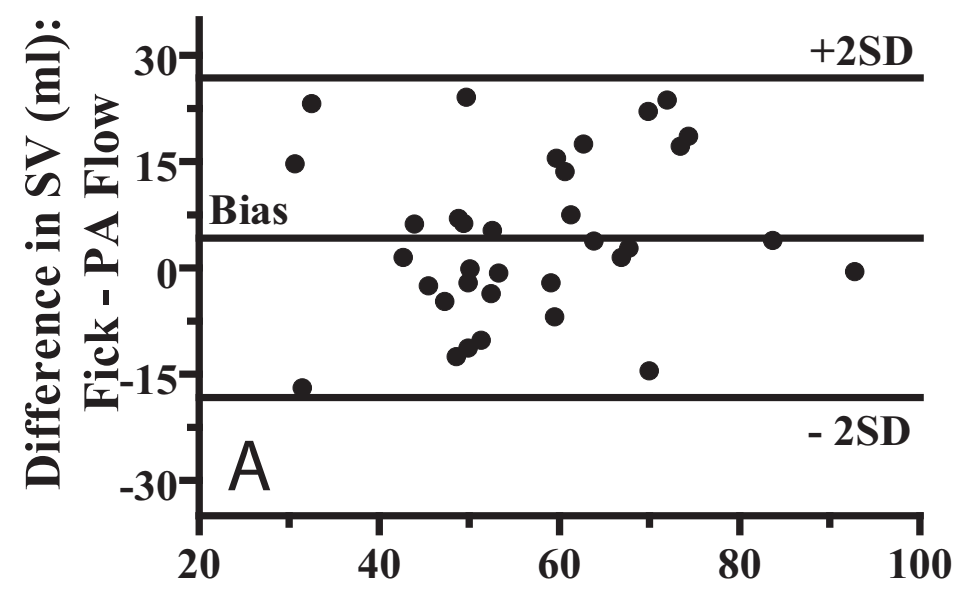

Mean SV (ml): Fick and PA Flow
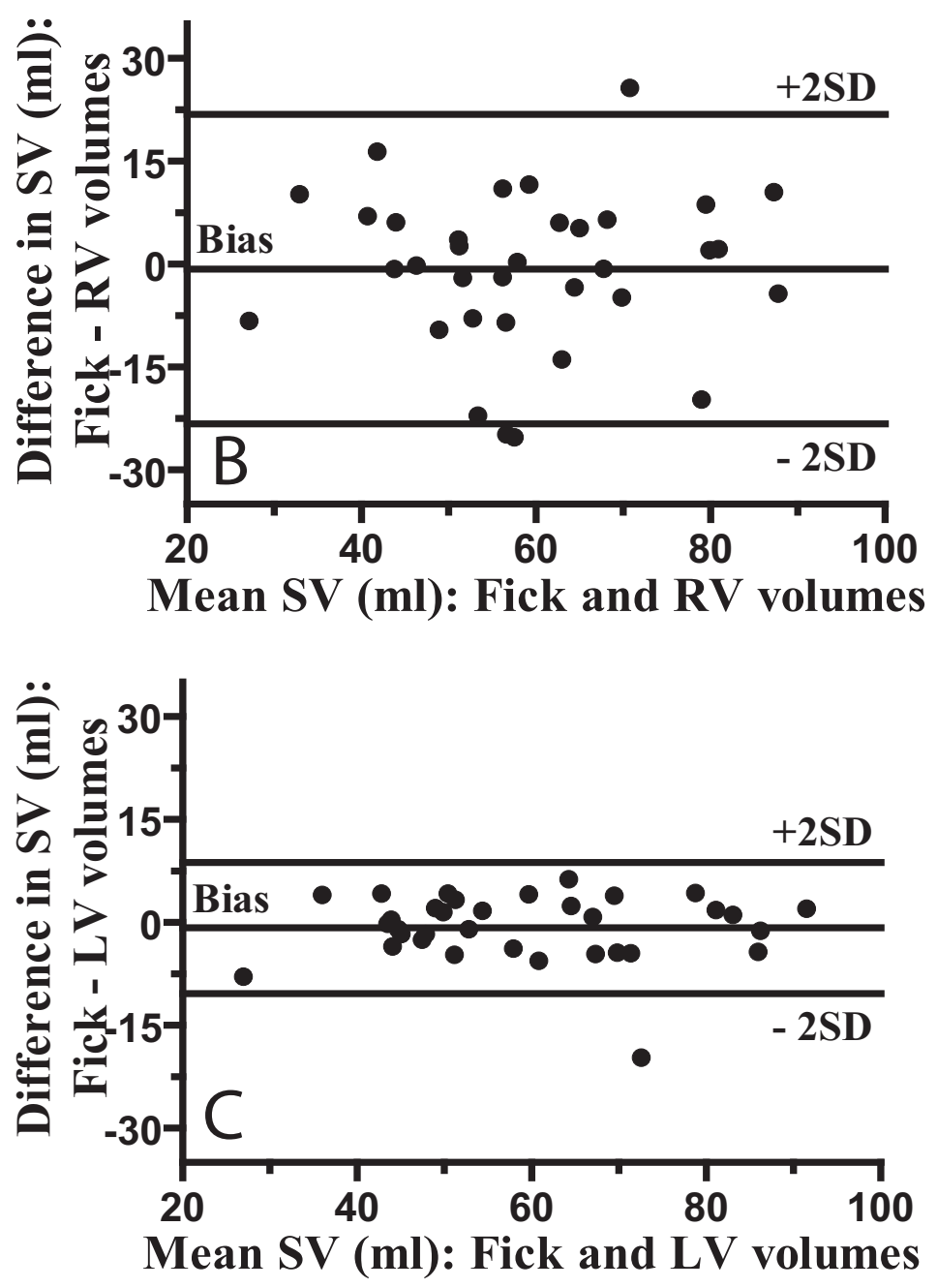

Figure 5

Bland-Altman plots shows degree of agreement between direct Fick and (a) PA flow, (b) RV volumes and (c) LV volumes in 34 PAH patients. Central line demonstrates bias; outer lines demonstrate upper and lower limits of agreement ( \pm 2 standard deviations [SD]) 
Table 2: Results of Bland-Altman analyses of CMR based measurements for stroke volume

\begin{tabular}{llllll}
\hline & Mean \pm SD & Bias & Precision & 95\% Limits of Agreement & Error (\%) \\
\hline PA Flow & $55.2 \pm 13.1$ & -4.2 & 11.48 & -18.3 to 26.8 & $42 \%$ \\
Aorta Flow* & $51.40 \pm 13.3$ & -2.3. & 3.85 & -9.8 to 5.2 & $14 \%$ \\
RV volumes & $59.5 \pm 15.7$ & -.75 & 11.49 & -23.3 to 21.8 & $39 \%$ \\
LV volumes & $59.6 \pm 16.2$ & -0.8 & 4.87 & -10.4 to 8.8 & $16 \%$ \\
\hline
\end{tabular}

NOTE. Values are $\mathrm{ml}, \mathrm{PA}=$ Pulmonary artery, $\mathrm{RV}=$ right ventricle, $\mathrm{LV}=$ Left ventricle. $*$ Aorta flow in nine patients.

The slight overestimation of SV by RV and LV volumes, $0.75 \mathrm{ml}$ and $0.8 \mathrm{ml}$ respectively, was not significant.

The SV assessed from the aortic flow (figure 6a) in the subset of 9 patients showed an tight relation with the Fickderived SV. The comparison with Fick was also made for the PA flow-derived SV, in the same subset of patients (figure $6 b)$.

Table 3 displays the intra-observer and inter-observer variability data. Intra-observer and interobserver bias for PA flow $(0.8-0.9 \mathrm{ml})$, as well as for Aorta flow $(0.7-1.3 \mathrm{ml})$, RV volumes (2.1-2.6 ml) and LV volumes (1.8-2.57 ml), were negligible. Intra-observer and inter-observer variability was sufficiently low for all different CMR methods for SV assessment, as shown by CoV values (2.7-13.3\%).

\section{Discussion}

To our knowledge this study is the first to assess the accuracy of SV derived non-invasively from PA flow, RV volumes, Aorta flow and LV volumes by comparing these values with stroke volume assessed by means of the invasive Fick method in a group of PAH patients.

Our results showed that SV derived non-invasively from PA flow and RV volumes were in poor agreement with the Fick-derived SV. By contrast SV measurement from LV volumes and aortic flow showed good agreement with Fick.

Although SV is highly variable in healthy subjects, an earlier study [18] revealed that SV is fixed in PAH and does not even change during exercise. Thus although the invasive and CMR measurements were not performed synchronously, we expect similar SV values for each patient. The direct Fick principle was chosen as the method of reference because it is considered as a standard for measuring SV in patients with PAH [14].

\section{SV assessment with PA flow}

SV determined from the pulmonary artery flow curve was poorly related to stroke volume assessed by means of Fick. There are several explanations for this discrepancy.
First, we observed non-laminar velocity profiles during systole (figure 1b) in the main PA of PAH patients, which can suggest turbulent or helical flow patterns. Phase-contrast measurements are optimized for laminar flow, whereas in turbulent flow patterns the precision of the CMR flow measurements declines $[19,20]$. In case of helical flow, motion in the non-velocity encoding directions may also lead to phase-shifts which are not related to through-plane flow.

Second, differences may result from cardiac right-to-left shunting, which causes underestimation of SV determination by PA flow. This is supported by our observation that SV using PA flow showed statistically significant underestimation of $4.2 \mathrm{ml}$; this underestimation disappeared by excluding those patients with a right-to-left shunt.

Another potential explanation is an inherent inaccuracy of the flow measurement by means of phase-contrast CMR. However, this explanation is unlikely, since in the subset of nine patients where aorta flow was also measured, a much better correlation with the direct Fick is found (figure 6). The accuracy of the aorta flow measurements for SV has already been validated by comparison with invasive SV measurements by Hundley et al [21] in 23 subjects.

\section{SV assessment with $R V$ volumes}

Several factors may explain the errors in the stroke volume measurements derived from the volumetric measures of the right ventricle. First, in the short axis view, in the most basal RV slice, with the RV outflow tract and the inflow region with the tricuspid valve, usually it is more complicated to define the RV contour than in the case of the left ventricle. Because of the large area of this basal slice, errors in choosing the exact RV contour can render inaccurate volume estimates and thus inaccurate SV measurements. Second, evaluation of right ventricular volumes in patients with PAH is difficult because of the complex anatomy of the right ventricle. Third RV endocardial boundary delineation is more complicated because of the considerably more trabeculation of the RV in comparison with the LV [22]. These factors may be improved with further software development to automate boundary detection and 

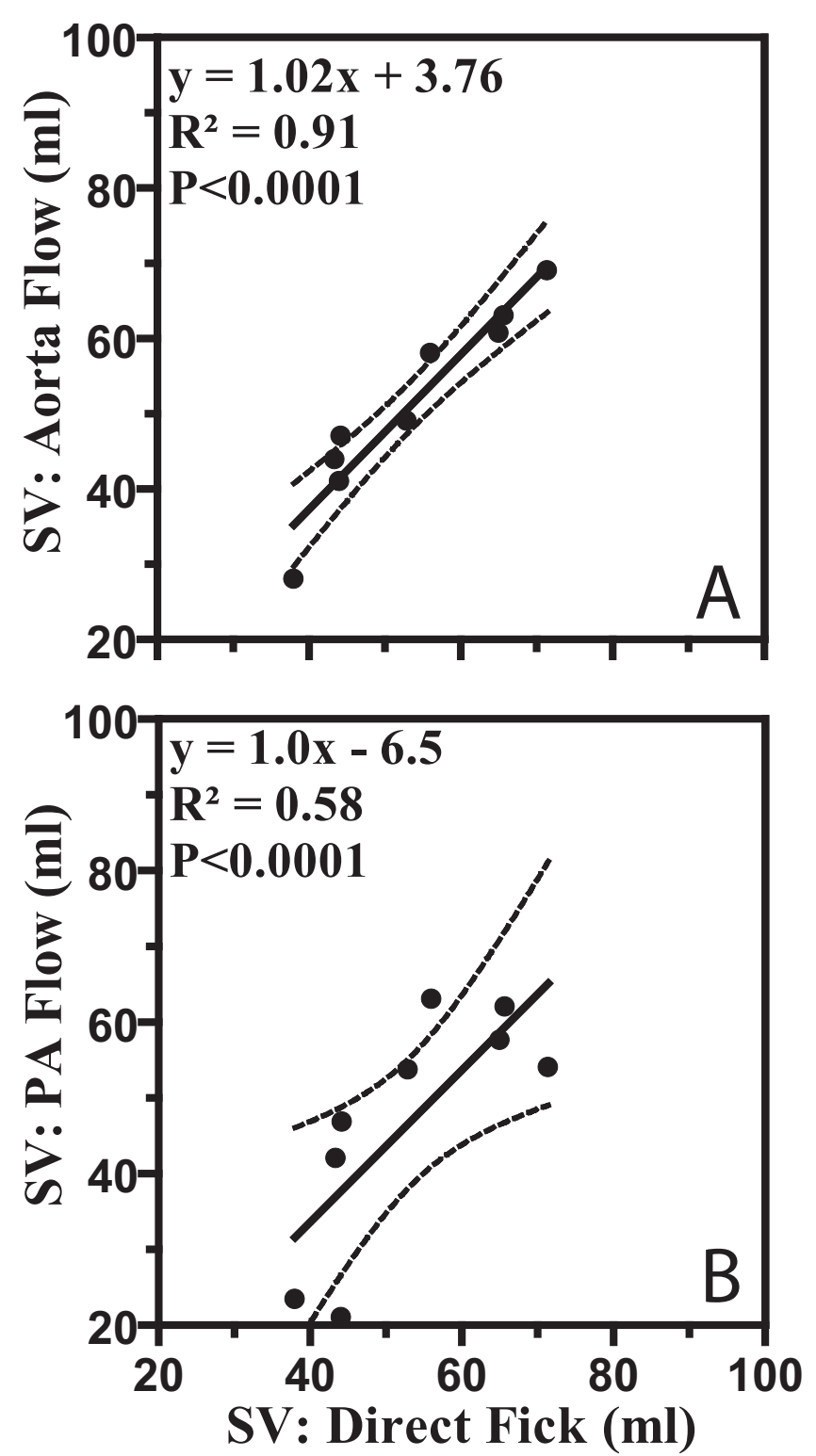

Figure 6

In a subset of nine patients both aorta and pulmonary flow was measured showing excellent correlation between the direct Fick and the (a) aorta flow in contrast to the direct Fick and the (b) pulmonary flow for stroke volume (SV). Dashed lines $=95 \% \mathrm{Cls}$.

also assist in the selection of the basal slice during analyses. Fourth, in patients with pulmonary hypertension tricuspid regurgitation (TR) is common. Thus, with considerable TR, the volumetric SV overestimates the actual SV [23], because it is impossible to differentiate between the volume that moves back through the tricuspid valves and forward though the pulmonary valves.

\section{SV assessment with $L V$ volumes}

The correlation between the SV derived from LV enddiastolic and end-systolic volumes and the SV from Fick was very tight (Figure $5 \mathrm{c}$ ). This is presumably so because for the LV it is rather easy to delineate the endocardial border and to select the basal slice.

Additionally there was no sign of systematic errors for low or high values indicating that the SV measure from LV volumes is valid for a wide range of SV Values. There were some high SV values in three patients with porto pulmonary syndrome which can be explained by the hyperdynamic circulation feature in these patients [24].

\section{Intra-observer and inter-observer variability}

The results of the current study indicated that the intraand interobserver variability of SV measurements using PA flow, aortic flow and LV volumes measurements were low, showing small differences and low coefficients of variability. A larger interobserver and intraobserver variability was shown for the RV volumes. This could be explained by factors mentioned above. Together with the poor agreement of RV-derived SV versus Fick, this advises against the use of RV volumes for SV determination.

\section{Clinical implications}

The accurate non-invasive SV assessment has clinical importance because monitoring of SV in PAH patients now becomes feasible as a routine method for monitoring the effects of medical treatment and the follow-up of patients non-invasively. The results of this study have important implications for PAH patients. The SV measurement using PA flow is less accurate in comparison to the SV derived from LV volumes and aorta flow. Therefore, LV volumes and aorta flow should be the methodology of choice in this patients group to measure SV accurately. Consequently, it is advisable to include in the CMR basic protocol a stack of contiguous short axis slices covering the full extend of the left ventricle, and an aortic flow measurement for accurate SV assessment.

\section{Limitations}

We acknowledge some limitations of our study including the fact that catheter studies and CMR examinations could not be performed simultaneously. However, the results of this study demonstrate that SV derived from LV volumes correlate well with measurements obtained using the direct Fick method, supporting earlier findings that stroke volume does not vary over a short period of time in $\mathrm{PAH}$, making this parameter of value to monitor PAH patients.

Furthermore we acknowledge the small sample size of the aorta flow measurements. 
Table 3: Intra-and interobserver variability of CMR based measurements for stroke volume.

\begin{tabular}{|c|c|c|c|c|}
\hline & \multicolumn{2}{|l|}{ Intraobserver Variability } & \multicolumn{2}{|l|}{ Interobserver variability } \\
\hline & $\operatorname{Bias}(95 \%$ Limits of agreement)* & CoV & Bias(95\% Limits of agreement) & CoV \\
\hline PA Flow & $\begin{array}{l}-0.8 \pm 1.7 \\
(-4.1 \text { to } 2.4)\end{array}$ & $3.1 \%$ & $\begin{array}{l}-0.9 \pm 2.2 \\
(-5.3 \text { to } 3.5)\end{array}$ & $4.0 \%$ \\
\hline Aorta Flow & $\begin{array}{l}-0.7 \pm 2.6 \\
(-5.4 \text { to } 5.0)\end{array}$ & $2.7 \%$ & $\begin{array}{l}-1.3 \pm 2.1 \\
(-4.1 \text { to } 5.2)\end{array}$ & $5.1 \%$ \\
\hline RV volumes & $\begin{array}{l}-2.1 \pm 5.2 \\
(-12.3 \text { to } 8.1)\end{array}$ & $8.7 \%$ & $\begin{array}{l}-2.6 \pm 7.9 \\
(-18 \text { to } 13.1)\end{array}$ & $13.3 \%$ \\
\hline LV volumes & $\begin{array}{l}-1.8 \pm 3.2 \\
(-8.0 \text { to } 4.4)\end{array}$ & $5.4 \%$ & $\begin{array}{l}-2.6 \pm 3.8 \\
(-10.1 \text { tot } 4.9)\end{array}$ & $6.4 \%$ \\
\hline
\end{tabular}

*Bias and limits of agreement were determined according to the Bland and Altman method. CoV = coefficient of variability, PA = Pulmonary artery, $\mathrm{RV}=$ right ventricle, $\mathrm{LV}=$ Left ventricle.

Finally, the present study does not provide conclusive evidence of the cause of the discrepancy between the PA flow and the direct Fick.

\section{Conclusion}

In patients with $\mathrm{PAH}$, taking the direct Fick as a standard invasive method, non-invasive derivation of SV should preferably be carried out using aortic flow or LV volumes. SV assessment with PA flow or RV volumes shows limited accuracy.

\section{Abbreviations}

CO: cardiac output; CoV: coefficient of variability; EDV: end diastolic volume; ESV: end Systolic volume; LV: left ventricular; CMR: cardiovascular magnetic resonance imaging; PA: pulmonary artery; PAH: pulmonary arterial hypertension; RHC: right heart catheterisation; RV: right ventricular; SV: stroke volume.

\section{Competing interests}

The authors declare that they have no competing interests.

\section{Authors' contributions}

GM participated in data collection, study design, data analysis and interpretation and manuscript preparation. JTM participated in data collection, study design, interpretation and manuscript revision. $\mathrm{AB}$ participated in interpretation and manuscript revision. PEP participated in manuscript revision. NW participated in interpretation and manuscript revision. AVN participated in study design, interpretation and manuscript revision. All authors read and approved the manuscript.

\section{References}

I. Torbicki A: Cardiac magnetic resonance in pulmonary arterial hypertension: a step in the right direction. Eur Heart J 2007, 28(10): I 187-9.

2. McLure LE, Peacock AJ: Imaging of the heart in pulmonary hypertension. Int J Clin Pract Suppl 2007:15-26.

3. van Wolferen SA, Marcus JT, Boonstra A, Marques KM, Bronzwaer JG, Spreeuwenberg MD, et al.: Prognostic value of right ventricu- lar mass, volume, and function in idiopathic pulmonary arterial hypertension. Eur Heart J 2007, 28(10): I250-7.

4. Roeleveld RJ, Vonk-Noordegraaf A, Marcus JT, Bronzwaer JG, Marques KM, Postmus PE, et al:: Effects of epoprostenol on right ventricular hypertrophy and dilatation in pulmonary hypertension. Chest 2004, I 25(2):572-9.

5. Kreitner KF, Kunz RP, Ley S, Oberholzer K, Neeb D, Gast KK, et al:: Chronic thromboembolic pulmonary hypertension - assessment by magnetic resonance imaging. Eur Radiol 2007, I7(I): || $|-2|$.

6. Marcus JT, Vonk NA, De Vries PM, van Rossum AC, Roseboom B, Heethaar RM, et al.: MRI evaluation of right ventricular pressure overload in chronic obstructive pulmonary disease. J Magn Reson Imaging 1998, 8(5):999-I005.

7. Kondo C, Caputo GR, Semelka R, Foster E, Shimakawa A, Higgins CB: Right and left ventricular stroke volume measurements with velocity-encoded cine MR imaging: in vitro and in vivo validation. AJR Am J Roentgenol I99|, I57(I):9-16.

8. Firmin DN, Nayler GL, Klipstein RH, Underwood SR, Rees RS, Longmore DB: In vivo validation of MR velocity imaging. J Comput Assist Tomogr 1987, II (5):75I-6.

9. Caputo GR, Kondo C, Masui T, Geraci SJ, Foster E, O'Sullivan MM, et al.: Right and left lung perfusion: in vitro and in vivo validation with oblique-angle, velocity-encoded cine MR imaging. Radiology 1991, I 80(3):693-8.

10. Bogren HG, Buonocore MH: Complex flow patterns in the great vessels: a review. Int J Card Imaging 1999, I5(2): 105-13.

II. Bogren HG, Klipstein RH, Mohiaddin RH, Firmin DN, Underwood SR, Rees RS, et al.: Pulmonary artery distensibility and blood flow patterns: a magnetic resonance study of normal subjects and of patients with pulmonary arterial hypertension. Am Heart J 1989, II 8(5 Pt I):990-9.

12. Okamoto M, Miyatake K, Kinoshita N, Sakakibara H, Nimura Y: Analysis of blood flow in pulmonary hypertension with the pulsed Doppler flowmeter combined with cross sectional echocardiography. Br Heart J 1989, 5 I (4):407-15.

13. Reuben SR, Swadling JP, de Lee GJ: Velocity profiles in the main pulmonary artery of dogs and man, measured with a thinfilm resistance anemometer. Circ Res 1970, 27(6):955-I00I.

14. Hoeper MM, Maier R, Tongers J, Niedermeyer J, Hohlfeld JM, Hamm $M$, et al: Determination of cardiac output by the Fick method, thermodilution, and acetylene rebreathing in pulmonary hypertension. Am J Respir Crit Care Med 1999, 160(2):535-4I.

15. Chernobelsky A, Shubayev O, Comeau CR, Wolff SD: Baseline correction of phase contrast images improves quantification of blood flow in the great vessels. J Cardiovasc Magn Reson 2007, 9(4):68I-5.

16. Marcus JT, Gotte MJ, DeWaal LK, Stam MR, Geest RJ Van der, Heethaar RM, et al.: The influence of through-plane motion on left ventricular volumes measured by magnetic resonance imaging: implications for image acquisition and analysis. J Cardiovasc Magn Reson 1999, I(1): I-6. 
17. Bland JM, Altman DG: Statistical methods for assessing agreement between two methods of clinical measurement. Lancet I(8476):307-I0.

18. Holverda S, Gan CT, Marcus JT, Postmus PE, Boonstra A, VonkNoordegraaf $A$ : Impaired stroke volume response to exercise in pulmonary arterial hypertension. J Am Coll Cardiol 47(8): $1732-3$.

19. Bakker CJ, Hoogeveen RM, Viergever MA: Construction of a protocol for measuring blood flow by two-dimensional phasecontrast MRA. J Magn Reson Imaging 1999, 9(1): I 19-27.

20. Evans AJ, Blinder RA, Herfkens RJ, Spritzer CE, Kuethe DO, Fram EK, et al.: Effects of turbulence on signal intensity in gradient echo images. Invest Radiol 1988, 23(7):5। 2-8.

21. Hundley WG, Li HF, Hillis LD, Meshack BM, Lange RA, Willard JE, et al.: Quantitation of cardiac output with velocity-encoded, phase-difference magnetic resonance imaging. Am J Cardiol 75(I7): I250-5.

22. Grothues F, Moon JC, Bellenger NG, Smith GS, Klein HU, Pennell DJ: Interstudy reproducibility of right ventricular volumes, function, and mass with cardiovascular magnetic resonance. Am Heart J 2004, I 47(2):2 I 8-23.

23. Hoeper MM, Tongers J, Leppert A, Baus S, Maier R, Lotz J: Evaluation of right ventricular performance with a right ventricular ejection fraction thermodilution catheter and MRI in patients with pulmonary hypertension. Chest 200I, 1 20(2):502-7.

24. Hoeper MM, Krowka MJ, Strassburg CP: Portopulmonary hypertension and hepatopulmonary syndrome. Lancet 363(94|9): $|46|-8$.

Publish with Biomed Central and every scientist can read your work free of charge

"BioMed Central will be the most significant development for disseminating the results of biomedical research in our lifetime. "

Sir Paul Nurse, Cancer Research UK

Your research papers will be:

- available free of charge to the entire biomedical community

- peer reviewed and published immediately upon acceptance

- cited in PubMed and archived on PubMed Central

- yours - you keep the copyright

Submit your manuscript here:

http://www.biomedcentral.com/info/publishing_adv.asp
BioMedcentral 